Cell Membrane Penetration without Pore Formation :

Chameleonic Properties of Dendrimers in Response to

Hydrophobic and Hydrophilic Environments

\author{
de Luca, Sergio
}

2020-07

de Luca , S , Seal , P , Parekh , H S , Tupally , K R \& Smith , S C 2020 , ' Cell Membrane

Penetration without Pore Formation: Chameleonic Properties of Dendrimers in Response to Hydrophobic and Hydrophilic Environments ' , Advanced theory and simulations , vol. 3 , no. 7 , 1900152 . https://doi.org/10.1002/adts.201900152

http://hdl.handle.net/10138/330533

https://doi.org/10.1002/adts.201900152

acceptedVersion

Downloaded from Helda, University of Helsinki institutional repository.

This is an electronic reprint of the original article.

This reprint may differ from the original in pagination and typographic detail.

Please cite the original version. 


\title{
Cell membrane penetration without pore formation: chameleonic properties of dendrimers in response to hydrophobic and hydrophilic environments
}

\author{
Sergio de Luca ${ }^{1}$, Prasenjit Seal ${ }^{2, *}$, Harendra S. Parekh ${ }^{3}$, Karnaker R Tupally ${ }^{3}$ \& Sean C. Smith ${ }^{1, *}$ \\ ${ }^{1}$ Integrated Materials Design Laboratory, Department of Applied Mathematics, Research School of \\ Physics, Australian National University, Acton, ACT 2601, Australia. \\ ${ }^{2}$ Department of Chemistry, University of Helsinki, P.O. Box 55 (A.I. Virtasen aukio 1), Helsinki \\ 00014, Finland. \\ E-mail: prasenjit.seal@helsinki.fi \\ ${ }^{3}$ School of Pharmacy, The University of Queensland, Brisbane, QLD 4072, Australia. \\ E-mail: sean.smith@anu.edu.au
}

\begin{abstract}
The mechanism by which cell-penetrating peptides and antimicrobial peptides cross plasma membranes is unknown, as is how cell-penetrating peptides facilitate drug delivery, mediating the transport of small molecules. Once non-disruptive and non-endocytotic pathways are excluded, pore formation is one of the proposed mechanisms, including toroidal, barrel-stave, or carpet models. Spontaneous pores have been observed in coarse-grained simulations and less often in molecular dynamics simulations. While pores are widely assumed and inferred, there is no unambiguous experimental evidence of the existence of pores. Some recent experimental studies contradict the mechanistic picture of pore formation, however, highlighting the possibility of a direct translocation pathway that is both non-disruptive and non-endocytotic.

In this work, we propose a model for peptide (linear and dendritic) translocation which does not require the presence of pores and which potentially accords with such experiments. We suggest that a charged peptide, as it experiences an increasingly hydrophobic environment within the membrane surface, can utilize a proton chain transfer mechanism to shed its protons to counter ions or potentially phospholipid head groups in the membrane skin region, thereby becoming compatible with the hydrophobic interior of the membrane. This increases the likelihood to move into the highly hydrophobic core of the membrane and ultimately reach the opposite leaflet to re-acquire protons again, suggesting a potential "chameleon" mechanism for non-disruptive and nonendocytotic membrane translocation. Our molecular dynamics simulations reveal stability of peptide bridges joining two membrane leaflets and demonstrate that this can facilitate crossmembrane transport of small drug molecules.
\end{abstract}


Antibiotic-resistant bacteria constitute a significant public health problem ${ }^{1,2}$. Antimicrobial peptides (AMPs) - a class of small ( $\leq 50$ residues), cationic or amphiphilic compounds - are amongst the most promising solutions currently known ${ }^{3}$. Cell-penetrating peptides (CPPs) are a similar class of peptides, important for their binding - usually via covalent conjugation through a disulfide bridge or by means of non-covalent interactions ${ }^{4}-$ and delivery of drugs into cells for therapeutic applications $^{5-7}$. Both CPPs and AMPs interact strongly with the membrane surface, penetrating or altering its permeability via a mechanism which is not well understood ${ }^{8,9}$, even after a plethora of experimental and simulation studies.

Atomistic molecular dynamics (MD) can be applied to investigate certain aspects of the uptake mechanisms. Despite its limitations in terms of accessible spatial and temporal scales, MD has the potential to provide unique insights. Several studies have converged, via observations or deductions, to infer the formation or existence of pores in the membrane $\mathrm{e}^{10-14}$. Pores have been observed in coarse-grained simulations ${ }^{15,16}$, and in molecular dynamics ${ }^{17,18}$. A spontaneous translocation of a TAT peptide was observed in one MD study ${ }^{19}$ and explained in terms of pore formation. However, subsequently this result was found to have originated from incorrect use of electrostatic interactions ${ }^{14}$. When a phosphatidylcholine (POPC) membrane is exposed to a CPP such as maculatin 1.1, dye leakage was observed after $\sim 10$ minutes. This result led the authors of the study to infer a physical mechanism based on membrane disruption or pore formation ${ }^{20}$. Binding energies of pore formation with alamethicin and melittin showed preference for cylindrical $^{21}$ and toroidal ${ }^{22}$ pores, in accord with molecular dynamics results ${ }^{23}$.

In contrast to the above picture, some experiments do not support the presence of pores. Considering that cationic peptides should interact more strongly with negatively charged compounds, one would have expected pore formations with a CPP oligoarginine peptide interacting with negatively charged lipids. Interestingly, a leakage was observed, but not with an ion-conductive structure ${ }^{24}$, which would have been expected in presence of holes or channels. Trans-activating transcriptional (TAT) peptide penetration has been observed in the absence of endocytosis, with the additional discovery that added fluorophores were not taken together with the TAT peptide - implying the unlikelihood of formation of nonselective pores ${ }^{25}$. This result suggested the possibility of a hitherto unknown direct translocation mechanism ${ }^{7,24-26}$. Effects on lipid bilayers of the TAT peptide derived from the HIV-1 virus TAT protein were investigated with low-angle X-ray scattering and MD, indicating that the TAT peptide binds to the neutral dioleoylphosphocholine (DOPC) and furthermore its positively charged guanidinium components 
of arginines get very close to the center of the bilayer ${ }^{27}$. Yet despite this no pore was observed: with MD a spontaneous water pore was generated only after constraining the peptides at $5 \AA$ away from the bilayer center. A microsecond-long simulation with antimicrobial peptide CM15 interacting with a POPC bilayer recorded significant membrane perturbations from the CM15 peptide, but pores were not detected ${ }^{28}$.

To shed more light on the issues, it is useful to summarize some of the physical membrane and peptide properties that can affect their membrane interactions. With arginine-rich penetrating peptides, a concentration threshold was discovered to be necessary for efficient translocation, suggesting a cooperative action by the peptides ${ }^{18}$. In another study an aggregate involving at least three peptides was found to be required for pore formation ${ }^{29}$. Peptide structure appears to be related to the pore species ${ }^{23}$, yielding specific peptide-pore pairing. The length and its ratio with the membrane thickness was shown to be more important than its helicity in the interactions within the membrane environment ${ }^{30}$. The membrane translocation abilities of oligo-arginines in living cells were found to depend on their chain length and peptide concentration, with nona- and deca-arginine attaining the best living cell transduction frequency ${ }^{6}$, also at lower concentrations ${ }^{31}$. Interestingly, a minimal number of six arginines per peptide in linear and/or dendritic arrangement was found necessary to enable transduction ${ }^{6}$.

With the above observational data in mind, the question arises as to whether the pore is a reliable model able to explain how short charged peptides translocate and permeabilize the membranes. It is germane to make three points in this regard: (i) over the past 20 years a pore has never been unequivocally observed experimentally. Assuming their existence, the current explanation is that they should have a very short lifetime, outside the probe capability of advanced techniques such as solid state $\mathrm{NMR}^{32}$. (ii) simulations reporting spontaneous peptide translocation via pore formation should be taken very cautiously, since there is a dramatic discrepancy between the experimental observation of pores and the frequency of pores observed in simulations. (iii) There does exist, as noted above, some experimental evidence suggestive of a non-endocytotic, non-disruptive translocation mechanism that obviates the need to postulate pore formation.

In this work we have undertaken simulations that lead us to propose a mechanism for peptide translocation of membranes without the presence of pores, disruptive events, or endocytotic processes. The model explains how a CPP acts as a small drug deliverer and accords naturally with two recent experimental results that are in striking contradiction with the pore formation model. Our main hypothesis is that the cationic peptide can be readily deprotonated when 
interacting with the membrane just inside its surface, becoming more hydrophobic and increasing the likelihood to penetrate the strongly hydrophobic core of the membrane. The peptide behaves as an hydrophobic-hydrophilic chameleon, losing and then reacquiring protons as needed to minimize its free energy within the environment. Free energy, or potential of mean force (PMF), profiles calculated for peptide translocation through the membrane show that when the peptide is deprotonated the PMF barrier is much smaller than that for translocation of its charged counterpart. This confirms that the deprotonation makes the peptide much more compatible with the strongly hydrophobic membrane environment. We implemented QM calculations to reveal that arginine (Arg) guanidinium groups, when encountering an hydrophobic environment, can effectively donate a proton via a chain transfer mechanism to a water environment several angstroms away with a solvated counter ion. Since the stabilization of the proton by the counterion $\left(\mathrm{Cl}^{-}\right.$in this case) is electrostatic rather than via a specific bond, the result carries the implication that hydrophilic phospholipid head groups could potentially also play such a stabilization role.

Further, our simulations reveal that a trans-membrane bridging dendrimer formed in this way is not only stable, but provides a low energy pathway for trans-membrane migration of small drug molecules, exemplified in our study by the anticancer drug 5-Fluro-Uracil (5FU).

Following methodology below, we present our simulation results and discuss the proposed "chameleon" model, with particular reference to two recent experimental studies that provide striking accord with its implications: one concerning TAT peptide membrane penetration and a second concerning transdermal delivery of a small (130 Da) antimetabolite drug.

\section{Methods}

Molecular dynamics simulations (MD) were performed using NAMD $2.10^{33}$. The CHARMM allatom force field ${ }^{34}$ was used to parametrize the molecular compounds under investigation, i.e., the membrane, the peptides, and the small anti-cancer drug 5-fluorouracil (5-FU). CHARMM has been extensively validated and employed to study structure and dynamics of membranes ${ }^{35}$ and proteins $^{36-38}$. We parameterized the amino acidic residues of our peptides using the input parameter file par-all36-prot ${ }^{34,39}$. The lipid membrane was downloaded from the web-based graphical interface CHARMM-GUI ${ }^{40}$ and parameterized with the CHARMM C36 force field for lipids ${ }^{39,41}$. The CHARMM-GUI input generator has been systematically tested in NAMD ${ }^{42}$, and implements several input generators for biological applications, among which the module membrane 
builder $^{35,43,44}$, which we have used.

To parameterize 5-FU we used the CHARMM compatible force field CHARMM General force field, or $\mathrm{CGENFF}^{45}$. Partial charges and parameters were downloaded from the CGENFF webserver ${ }^{45}$, providing atom typing and assignments by chemical analogy ${ }^{46,47}$ for atomic charges, bond, dihedral and improper polymer parameters. The 5-FU molecule was built with the 3D builder in MAESTRO Schrodinger 2.8.013. Peptides were constructed patching the amino acids from the CHARMM protein library ${ }^{36,37}$ following the NAMD tutorial procedure ${ }^{48}$ for merging and patching the molecular compounds. Details for the 5-FU and peptide construction and validation have been detailed in our previous work ${ }^{49,50}$. Here we only mention that we validated the interactions between polymers and the small anticancer drug $5-\mathrm{FU}^{49}$ by constructing several peptide dendrimers and demonstrating that the radius of gyration as a function of monomer size followed with good agreement a trend observed for similar sized polymeric systems ${ }^{51,52}$. This validation is further supported by the qualitative agreement found between our molecular dynamics results ${ }^{49}$ and the experimental results of the peptide dendrimer R4, R8, and R16 enhanced solubility effects for 5$\mathrm{FU}^{53}$.

Peptides, 5-FU and membrane are arranged in a cubic box of size $\sim 6 \times 6 \times 10 \mathrm{~nm}^{3}$ using $\mathrm{PACKMOL}^{54}$ and solvated with the solvate VMD plugin ${ }^{55}$ using the water model TIP3P ${ }^{56}$. The $\mathrm{Cl}^{-}$ ions are added to make the systems electro-neutral. Each system contains roughly 34000 total number of atoms with $\sim 10000$ water molecules each. In the initial configuration, peptide and 5-FU are positioned in water, such that their minimum separation from the cubic box border and among themselves is no less than $\sim 1 \mathrm{~nm}$ in each direction. We assume the water solution at $\mathrm{pH}=7$, with neutral DOPC and charged octaarginne (linear) and R8 (dendritic), at the beginning.

The peptides employed in the experimental study that we allude to in the discussion below are R4, which has $C$-to- $N$ sequence Gly-Lys-(Arg) $)_{2}$; R8 with $8^{+}$and Gly-Lys-(Lys) $)_{2}-(\operatorname{Arg})_{4}$; and R16 with $16^{+}$and Gly-Lys-(Lys) $)_{2}-(\text { Lys })_{4}-(\text { Arg })_{8}$, with masses 5151084 , and 2223 Da, respectively ${ }^{49,50,53}$. In this work, we employ R8 and octaarginine, since R8 was the best performed as a transdermal permeability enhancer for $5-\mathrm{FU}^{53}$.

Fig. 1 plots a top view of the system, with the membrane phosphorous atoms colored in blue, a lateral view (center), and a 3D view (right) with the simulation box in blue, the peptide in its bridge configuration (green, discussed further below), and a few 5-FU molecules (yellow) immersed in water (red and white). Fig. 2(left) plots the chemical structure of a single membrane DOPC phospholipid and 5-FU on the right. Fig. 3(left) plot R8 and octaarginine (right). All the 
pictures are visualized with $\mathrm{VMD}^{55}$.

The peptide was first minimized and equilibrated in an aqueous solution, without the membrane. Then the full system was assembled, minimized and equilibrated for $50 \mathrm{~ns}$ in NPT conditions at $\mathrm{T}=$ $298 \mathrm{~K}$ and pressure $1 \mathrm{bar}$, as for production runs. The van der Waals interactions are truncated at the cut off distance of $1.2 \mathrm{~nm}$ with a smooth switching function ${ }^{33}$. All pairs of bonded atoms that match the $1-4$ criteria are excluded ${ }^{33}$. Periodic boundary conditions are used in each direction. The Particle Mesh Ewald ${ }^{57}$ method is used to calculate the full electrostatic interactions with a grid spacing of $1 \AA$, computed every 4 steps. The temperature is kept constant with the Langevin thermostat and 1 fs time

step was used to integrate the equations of motion. The velocity-Verlet integration scheme ${ }^{58}$ is used to evolve the atom positions. Data analysis has been conducted with TCL scripts.
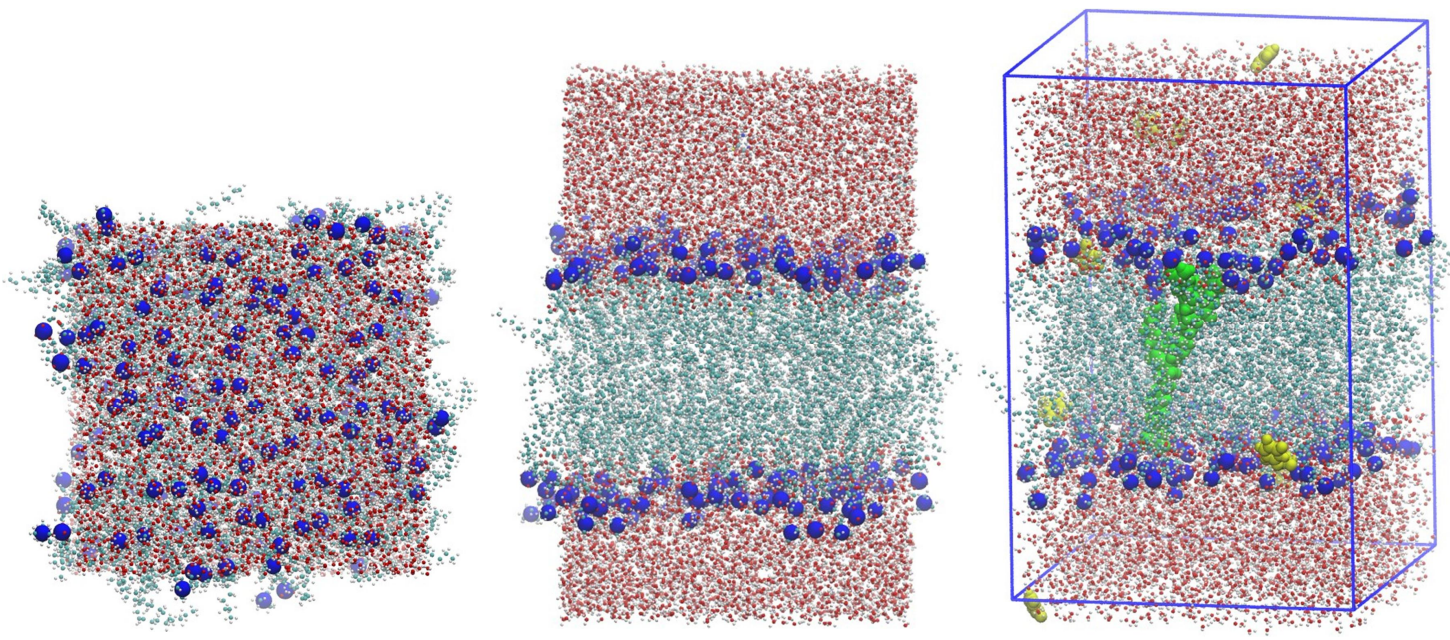

Fig. 1 (Left) DOPC membrane top view visualized with $\mathrm{VMD}^{55}$. Nitrogen and phosphorous atoms are plotted in blue. (Center) Section view of the system, with water and the DOPC membrane. (Right) R8 bridge configuration (green), with 5FU (yellow)
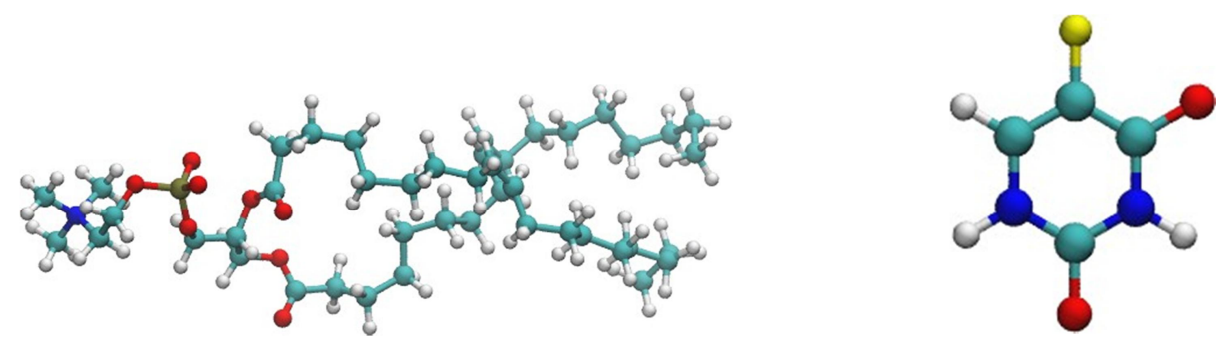

Fig. 2 (Left) Single DOPC phospholipid downloaded from CHARMM-GUY graphical interface ${ }^{40}$, with the hydrophobic fatty acid tail group and head groups colored according to the CHARMM atom type, carbon with cyan, phosphorus with brown, hydrogen with white, oxygen with red, and nitrogen 
with blue. (Right) Chemical structure of 5-Fluorouracil, with fluorine colored in yellow. Pictures are visualized with $\mathrm{VMD}^{55}$
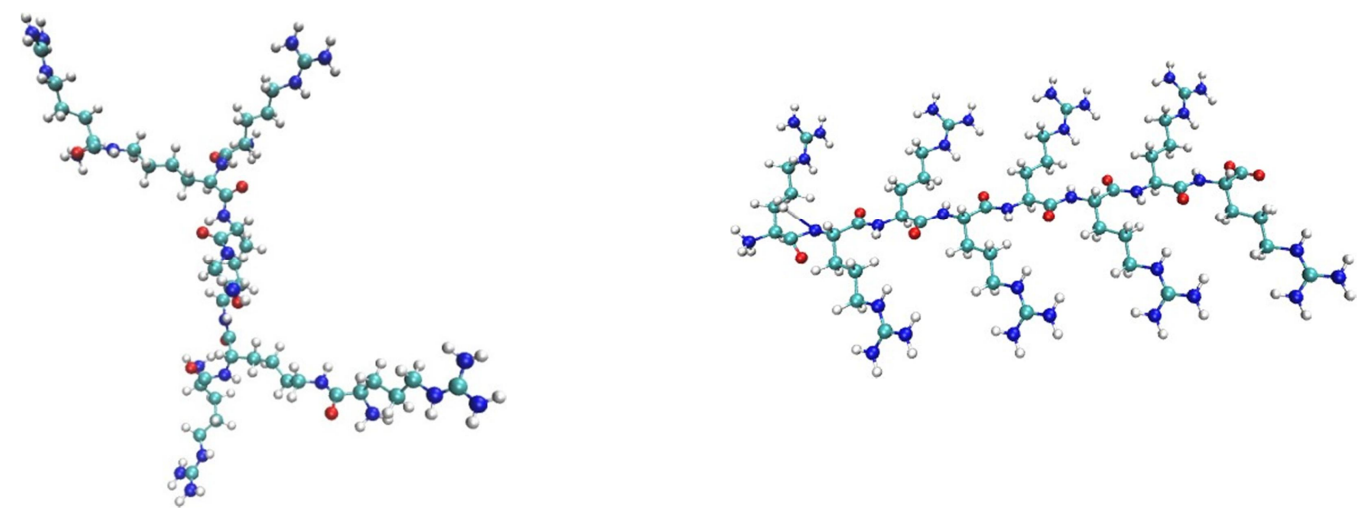

Fig. 3 (Left) Chemical structure of R8 and (Right) octaarginine. Visualization with VMD ${ }^{55}$ embedded in $\mathrm{VMD}^{55}$ and python

We model the membrane with dioleylphosphatidylcholine (DOPC), one of the most typical cell membrane lipids with one tail composed of eighteen carbon atoms and one double-bond in the middle of each chain (unsaturation), and with a transition temperature of $-16.5{ }^{\circ} \mathrm{C}^{59}$, therefore existing in the fluid like liquid crystalline state $\left(\mathrm{L}_{\alpha}{ }^{60}\right)$ at room temperature.

The surface, or outer leaflet, of mammalian cell membranes is composed of electrically neutral, zwitterionic phospholipids, hence DOPC is the appropriate model membrane in our study ${ }^{61}$. To validate our implementation, we compare the DOPC area per lipid of the CHARMM model, $A=69.7$ $\AA^{235,39-44}$ with our MD measurements. The number of DOPC phospholipids is our system is $\mathrm{N}_{D}=104$ with 52 molecules per leaflet, with the surface area per lipid equal to the averaged area of the lateral dimensions ( $x$ and $y$ ) of the simulation box divided by $\mathrm{N}_{D} / 2$. The time series of the area per lipid is plot in Fig. 4 (left), and the relative histogram on Fig. 4 (right). The result shows that the expected value $A=69.7 \AA^{2}$, evidenced with the vertical bar in Fig. 4 (right), is within the range of our MD measurement.

In our MD results we have not observed any event, neither a 5-FU entrance inside the membrane, nor a peptide translocation, on time scales of the order of $1 \mu \mathrm{s}$. Given the slow diffusion of solutes in the membrane $\left(\sim 10^{8} \mathrm{~cm}^{2} \mathrm{~s}^{-1}\right)^{62}$ advanced sampling techniques in molecular simulation can be utilized to assess the free-energy profile across the membrane, and to examine events which cannot be observed with the time scales available, such as translocation events. 

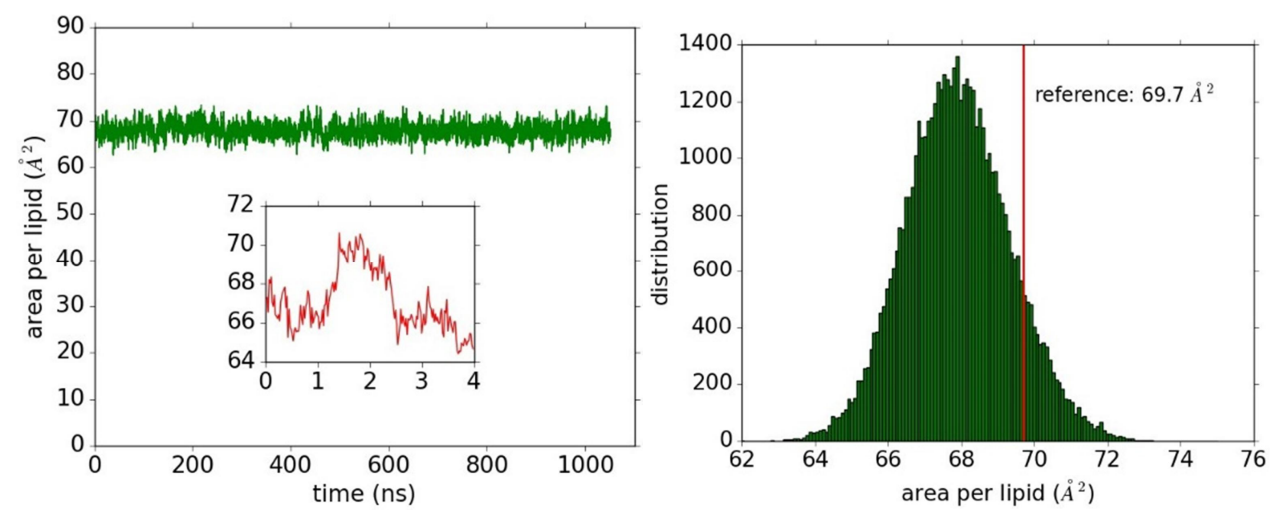

Fig. 4 (Left) DOPC surface area per lipid with time. The inset units are the same as the main graph. (Right) Histogram of the instantaneous area per lipid with 150 bins, with the vertical bar reporting the value $A=69.7 \AA^{2}$ suggested by the literature ${ }^{35,39-44}$

Free energy calculations are made using the $\mathrm{ABF}^{63,64}$ method, with its implementation in $\mathrm{NAMD}^{33}$. We averaged for $40 \mathrm{~ns}$ in total for each of the 15 windows of width $2 \AA$, to cover a distance of $0-30 \AA$ across the reaction coordinate. We verified that after $20 \mathrm{~ns}$ from the beginning the PMF profile does not change. Hence, our PMF profile, which is averaged over the successive $20 \mathrm{~ns}$, is convergent. The bin count per window was larger than $10^{5}$.

The reaction coordinate employed to investigate the 5-FU permeation through the lipid bilayer is the distance of the permeant molecule center of mass and the bilayer center, projected onto the bilayer normal. The same reaction coordinate is applied when the 5-FU PMF is computed in presence of R8. The peptide is positioned perpendicularly to the two membrane leaflets, with 5-FU located at the initial configuration, in its assigned window and within $0.8-1 \mathrm{~nm}$ from the peptide, i.e., not too close to perturb the peptide bridge configuration, affecting the outcome, and not too far away such that 5-FU would simply abandon the peptide choosing a random direction along the slab. Note that the 5-FU is free to diffuse in its assigned parallel slab, that is, is free to diffuse in the hydrophobic environment along the $\mathrm{x}-\mathrm{y}$ directions. Note also that, after the peptide has been dragged into its bridge or ladder configuration, it is left free, that is, in our PMF computation, the whole system is free and nowhere are applied external constraining forces.

Conscious that the parametrized MD force-field will not effectively describe the propensity for proton shedding by the charged peptide, we have undertaken reduced-model quantum chemical calculations to explore this process as described in detail below. The quantum chemical calculations were carried out using B3LYP/6-31G(d) in n-hexane implicit solvent, employing the polarizable continuum model (PCM) using the integral equation formalism variant (IEFPCM) ${ }^{65}$ as implemented in Gaussian ${ }^{66}$ suite of programs. The implicit hydrophobic solvent, n-hexane, was 
used in our calculations to mimic the lipid environment within the membrane as discussed below.

\section{Results}

First, we study the behavior of a free peptide diffusing in water and in presence of a DOPC membrane. Almost immediately ( $\sim 50 \mathrm{~ns})$, the peptide interacts with the membrane and will not detach for the next $\sim 2 \mu \mathrm{s}$, suggesting very strong electrostatic interactions. We plot the distance between the R8 center of mass and the membrane surface center of mass in function of time in Fig. 5 (left). The membrane surface centre of mass is computed as the average center of mass (CM) position of the phospholipid's head nitrogen atoms. The plot indicates that the R8's CM remains prevalently under the external surface, but does not enter the hydrophobic core on the time scale examined.

Following the strategy outlined in the Introduction and Methods, we deprotonate R8 after $1.2 \mu \mathrm{s}$ passing from $\mathrm{Q}=+8$ e to $\mathrm{Q}=0$, and then continue the simulation for a further $\sim 800 \mathrm{~ns}$. The $\mathrm{R} 8$ membrane relative position is not affected, for $\mathrm{R} 8$ remains close to the bilayer surface without penetrating further. We use this average position, attained without applying any external constraint force, as the starting point for the calculation of the free energy profile for translocation. It is important to note that this procedure is different from the standard method to compute translocation free energies, where it is computed starting with the peptide located in water. However, since the proposed model involves a possible change of charge state once the peptide has diffused inside the leaflet this is a practical common starting point to consider the comparative free energy profiles with and without deprotonation. In Fig. 5 (right) the reaction coordinate crosses the membrane perpendicularly and the distance corresponding to $20 \AA$ in the picture is the $\mathrm{CM}$ position attained by the peptide after $1.2 \mu \mathrm{s}$ simulation time (the final part of the red profile in Fig. 5(left)). The zero point on the $\mathrm{x}$-axis corresponds to the center of the membrane. The PMF result in Fig. 5 (right), obtained with the $\mathrm{ABF}$ method $^{63,64}$, indicates $52 \mathrm{kcal} / \mathrm{mol}$ for R8 with $\mathrm{Q}=+8 \mathrm{e}$ ( $\mathrm{e}$ is the elementary electric charge, $1.6 \times 10^{-19}$ Coulomb). Computing the PMF starting from the same position but after deprotonating $\mathrm{R} 8$, the barrier is reduced a factor of $\sim 5$, indicating that the deprotonation strongly increases the peptide-membrane hydrophobic core affinity. In turn, the likelihood for the peptide to reach the opposite leaflet is dramatically increased, implying that the non-disruptive translocation may be a much more probable event than hitherto believed. It is interesting to note that the translocation free energy of a single arginine amino acid through a DPPC bilayer was found to be $13.3 \mathrm{kcal} / \mathrm{mol}^{67}$ (with the CHARMM forcefield ${ }^{34}$ ), very similar to 
the free energy for the whole deprotonated R8, which is made from eight amino acids, as shown in Fig.5(left) of $\sim 15 \mathrm{kcal} / \mathrm{mol}$.

The energetic cost to transfer positively charged Arg from water to the interior of a membrane suggests that this process should be highly unfavourable, in striking contrast with the experimental evidence that CPPs cross the membrane quite easily on a timescale of minutes ${ }^{68}$. Our model reconciles a non-disruptive penetration mechanism with the apparently modest translocation energy barrier inferred from experiment, suggesting that once the peptide is deprotonated the hydrophobic barrier is much lower than previously thought.
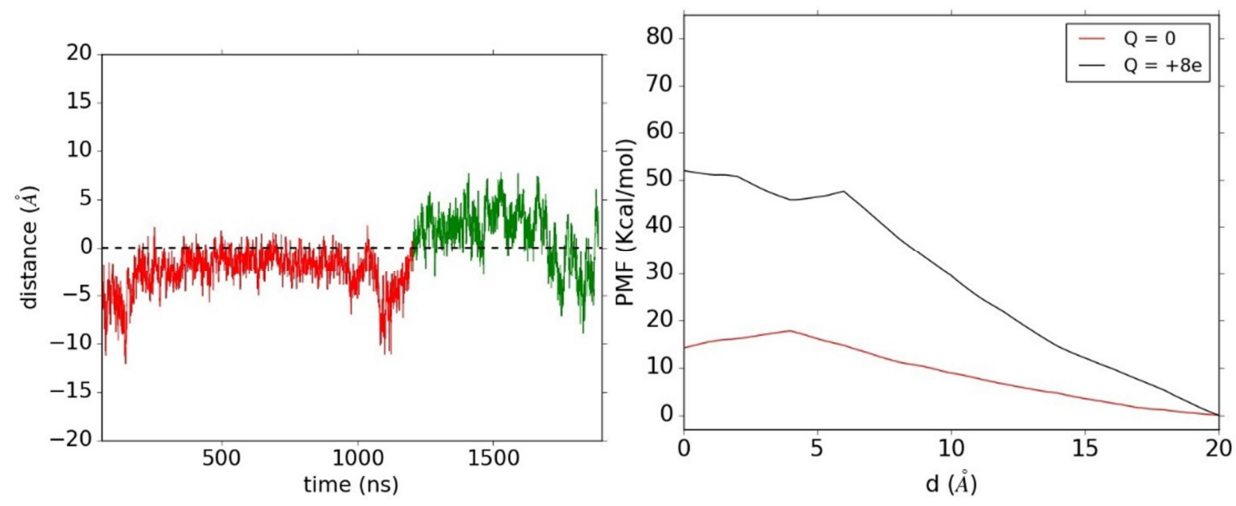

Fig. 5 (Left) Distance as a function of time between the center of mass of the peptide and the center of mass of the external surface of the membrane, computed averaging over the nitrogen atoms of its phospholipid headgroups. When $\mathrm{R} 8$ is protonated the time profile is plot with red, when it is deprotonated $(\mathrm{Q}=0)$ with green. (Right) $\mathrm{PMF}$ of the peptide translocation through the membrane. The black profile corresponds to the PMF of $\mathrm{R} 8$ with charge $\mathrm{Q}=+8 \mathrm{e}$, and the red profile plots the PMF of the deprotonated peptide. In both PMFs, the initial peptide position is the one attained at $1.2 \mu \mathrm{s}$ of the picture on the left.

We must point out that our deprotonation scheme is in contrast with most literature which states that Arg keeps its positive charges within the hydrophobic environment of the membrane ${ }^{69-73}$, with relatively few cases arguing for a possibility of both the protonated and neutral state ${ }^{74}$. The problem is still under debate ${ }^{9}$ and involves considerable complexity, given the influence that water defects and membrane deformation can have on the energetics of partitioning ${ }^{69,74}$. The pKa of Arg is predicted to remain relatively constant at least until it is already close to the hydrophobic centre of the membrane ${ }^{69,75}$, apparently belying the likelihood that the peptide could shed its protons as it starts to penetrate the hydrophobic region. This begs the mechanistic question as to how such proton loss might actually occur.

In considering the mechanism for proton loss by the peptide, we start with a simple question: would the proton be more stable (a) on Arg in the hydrophobic lipid environment or (b) out in the 
exterior skin region where there is water and counter-ions? Clearly, the latter environment should more readily stabilize and solvate the excess proton. However, once the peptide starts to penetrate the membrane interior there will be perhaps several ansgtroms separation distance out to the exterior skin region, so by what mechanism could a proton traverse the distance back to the more stable environment? Noting that the water concentration decreases steadily but does not become negligible until well into the membrane interior (see Fig. 9, left panel), we suggest it is entirely possible that water molecules can form a proton wire linking the two states. In this way, proton chain transfer - a phenomenon well known in other biological contexts such as the Green Fluorescent Protein photocycle ${ }^{76}$ - presents itself as a likely mechanism by which the peptide can effectively release its excess protons back out to the membrane skin region.

To explore this proposed mechanism in more detail, we have undertaken quantum chemical (QM) calculations on a representative model shown schematically in Fig. 6 below. Implicit solvent models implemented within the QM calculation allow the model to mimic an hydrophobic environment. In the current context, we wish to represent a transition from an hydrophobic environment further into the membrane to an hydrophilic environment in the exterior skin region. Since the implicit solvent model is applied to the whole system, we represent the hydrophilic exterior region by a water cluster, so that the interior of the cluster may reasonably approximate the hydrophilic environment in the exterior region. We include a $\mathrm{Cl}^{-}$counter ion within the water cluster to represent the fact that there are negatively charged species in the exterior skin environment (either counter ions or phospholipid head groups) that can electrostatically stabilize the hydrated proton in that region. For simplicity we use a single Arg residue in place of the dendrimer. With this model in mind, we postulate that the energy gradient for the overall charge transfer due to the change in hydrophobicity between the membrane interior and the membrane skin region could drive the proton chain transfer and thence provide the mechanism for deprotonation. 


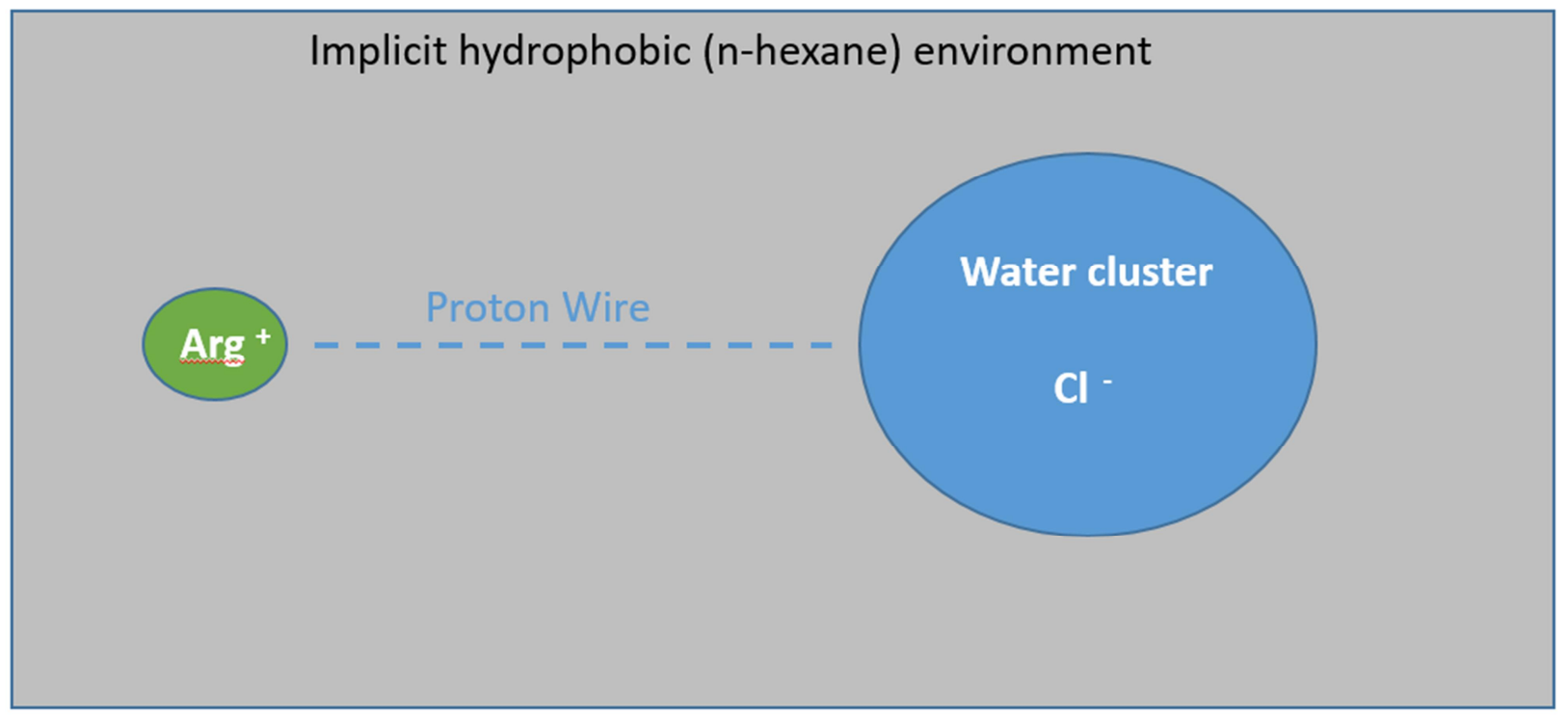

Fig. 6 Schematic of QM model

Following this strategy, the upper frame of Fig. 7 shows initial state for the proton transfer with the excess proton on the Arg residue, optimized at the B3LYP/6-31G(d) level of theory. The lower frame shows the final optimized state where the proton resides in the vicinity of the $\mathrm{Cl}^{-}$counter ion within the water cluster. Note that this final state involves a loose electrostatic stabilization of the proton by the counter ion rather than a dative bond; hence, even a phospholipid head group could potentially play such a role. In Fig.7, we have cropped extraneous atoms in the models and highlighted in white the atoms directly involved in this proton-chain transfer pathway for visual clarity; complete figures with atomic colour scheme are provided in the supplementary information (Figure S1). For this model, we included three bridging water molecules between Arg and the water cluster to exemplify a proton wire arrangement. To facilitate the calculations, we have constrained the positions of the $\mathrm{O}$ atoms of the bridging water molecules and water cluster, as well as the counterion. As mentioned earlier, an implicit hydrophobic (n-hexane) solvent model was applied, employing the polarizable continuum model (PCM) using the integral equation formalism variant $(\text { IEFPCM })^{65}$ as implemented in Gaussian ${ }^{66}$ suite of programs. The calculated energies reveal that the final state with the excess proton in the water cluster is $\sim 40 \mathrm{kcal}$ more stable than the initial state, confirming the expectation that there will be a significant energy gradient in favour of the proton transfer. 

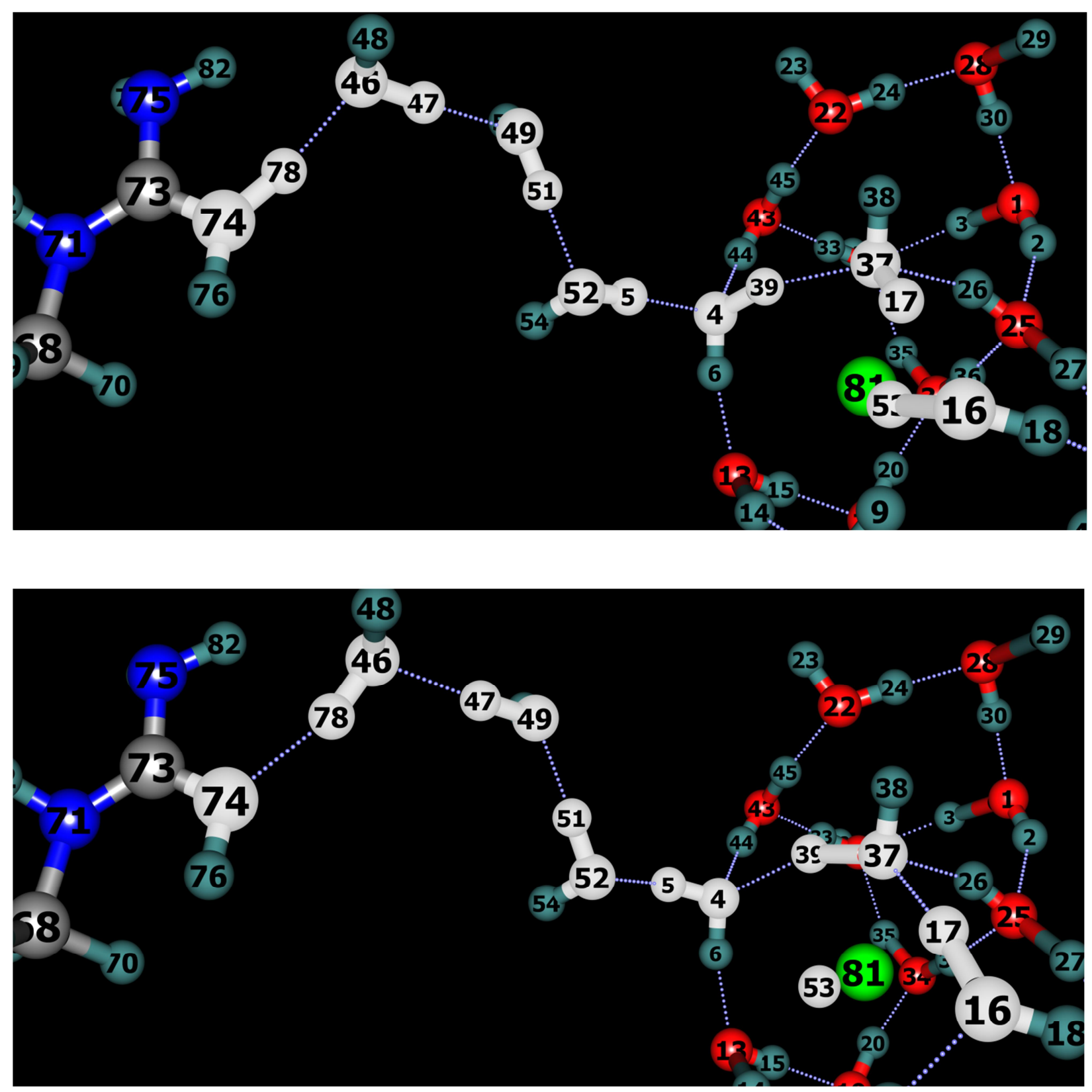

Fig. 7 Initial (upper frame) and final (lower frame) optimized QM model structures for proton chain transfer from protonated Arg. The white highlighted portion indicates the proton transfer pathway.

It remains to explore the energetics of the proton chain transfer. The complexity of this system prohibited successful utilization of the automatic minimum energy pathway searching method as implemented within Gaussian. In lieu thereof, we have used a simple approach to generate a 
coarse-grained pathway involving motions of the protons. We move protons $78,47,51,5,39,17$ and finally 53 in a manner that approximately maintains the $\mathrm{H}-\mathrm{O}-\mathrm{H}$ bond angles for the water molecules $\left(\sim 105^{\circ}\right)$ throughout the transfer and reaches the desired final structure where the proton resides inside the water cluster. We divided the path of each of the protons into 6 intervals, giving 6 points along the pathway of each. We implemented three different scenarios for collective motion of the protons that would result in an overall proton chain transfer from the protonated Arg to the water cluster. The first set of movements represents a "push" mechanism where the collective motions start from the first proton in the chain and propagate forward along the chain like falling dominoes. From point to point along the pathway, these motions can be described as follows:

1. Initial point

2. Move 78 by 1 step and compute energy.

3. Move 78 by a 2 nd step and move 47 by 1 step and compute energy.

4. Move 78 by a 3 rd step; move 47 by a 2 nd step; move 51 by 1 step and compute energy.

5. Move 78 by a 4th step; move 47 by a 3 rd step; move 51 by a 2 nd step and move 5 by 1 step and compute energy; and so on until the final state is reached.

The second set of movements represents a "pull" mechanism wherein the last proton in the chain moves first, initiating a cascade of (forward) movements that propagates back along the chain (i.e., the first proton in the chain is the last to start moving) until all protons have reached their final positions. The final set of movements is fully "synchronous", wherein each proton in the chain moves one increment at the same time as all the others. The specific H-bond lengths for these three different propagation scenarios are shown explicitly in Tables S1-S3 of the Supplementary Information together with the computed energies, which are plotted to yield the three corresponding potential energy profiles in Fig.8. Interestingly, we find that the "push" and "pull" mechanisms have similar energetic barriers and are much lower than that computed for the synchronous mechanism. Clearly, these pathways are far from optimized and we expect that a fully optimized pathway would have a significantly lower barrier. In any event, with barriers for the unoptimized push and pull mechanisms less than $30 \mathrm{kcal}$, the proton chain transfer pathway seems quite feasible. It goes also without saying that a plethora of such proton wire pathways would be available to the dendrimer given the fluidity of the water molecules in the system. 


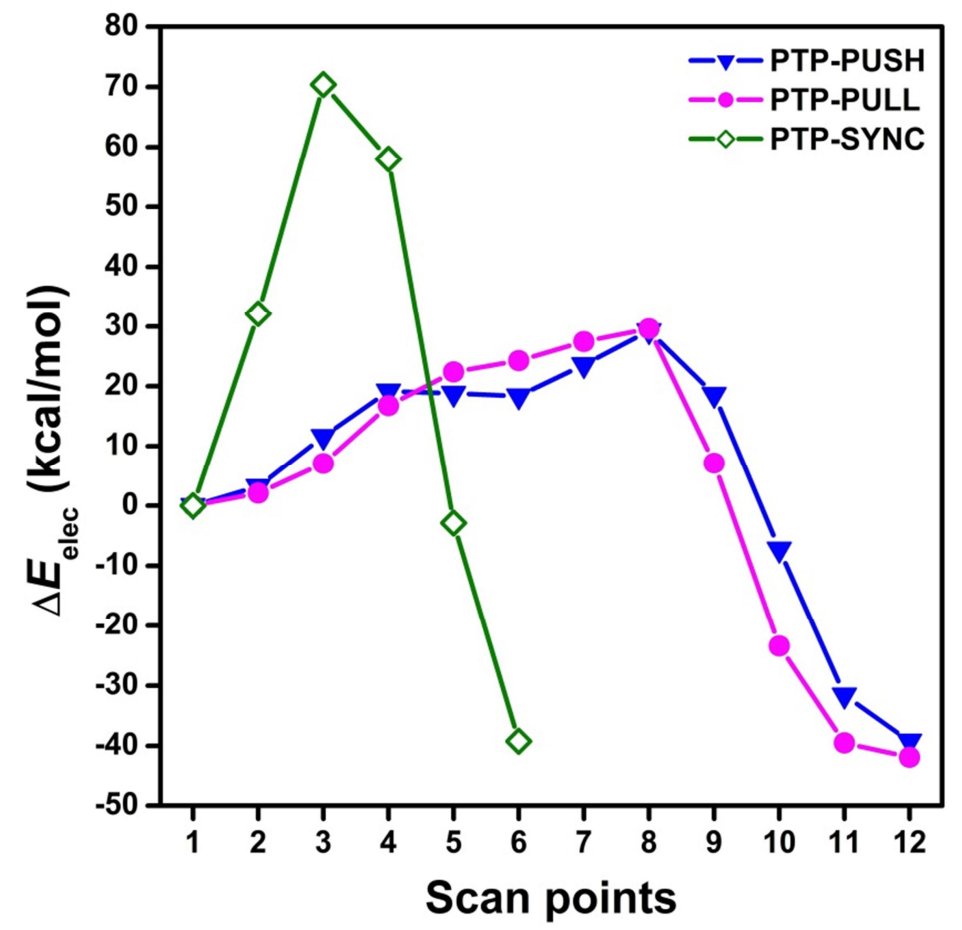

Fig. 8 Potential energy profile obtained from single-point calculations corresponding to all the mechanisms described in the text.

It will be apparent that the uncharged PMF profile in Fig.5 (red line, right frame) is based on an idealized model, whereby the dendrimer loses all its protons just after penetrating inside the membrane skin and then migrates into the lipid interior in an uncharged state. The implication of the quantum chemical model we have studied here is that the protons may be shed over a relatively short distance (i.e., a few Angstroms) as (i) the water concentration drops and (ii) the environment becomes more hydrophobic, creating the thermodynamic driver for the proton chain transfers to occur. Indeed, it may not even be necessary for all protons to be shed in order for the membrane penetration to become feasible. If one envisages all protons being shed progressively as the peptide penetrates into the membrane, then qualitatively the peptide PMF profile would start out along the fully-charged black line (right frame of Fig.5) and then progressively track across onto the uncharged red line as the protons are shed. We emphasize also that it is the proton chain-transfer mechanism - bridging the initial and final states of Fig.7 and enabling charge transfer over several Angstroms - that allows the energy difference between the two states to become an effective thermodynamic driver for the shedding of protons by the peptide. As noted above, earlier work ${ }^{69,75}$ involving $\mathrm{pKa}$ calculations has shown that there is no obvious driver for proton shedding if one only considers local transfer of a proton. 
It is instructive to compare our model with two experiments in order to tease out some of its implications. The first experiment employed neutron lamellar diffraction to investigate the interaction between a neutral DOPC membrane and TAT peptides ${ }^{7}$. A deeper insertion of TAT was observed in the hydrophobic core at depth $6 \AA$ from the center of the bilayer close to the double bond of fatty acyl chain ${ }^{7}$, leading the authors to suggest that TAT translocation may not depend on the electrostatic interactions between the ionic peptide and membrane groups interactions. Our model provides an easy answer here: that they are not important because the positive groups on the peptide lose their protons upon entry into the membrane, becoming more hydrophobic. Fig. 9(left) plots the mass density profile of the membrane and dendrimer atomic species. The profile is averaged over the whole simulation time in Fig. 5(left), including when R8 is deprotonated. Note that the dendrimer profile resembles a gaussian profile, analogous to that which has been found with a neutron diffraction experiment of the internalization of TAT peptide in DOPC phospholipid membrane ${ }^{77}$. Non-selective pore models, as already mentioned in the Introduction, should predict a roughly flat peptide mass density profile, that is, uniform across the membrane section. Our model, hence, provides a sensible explanation of the aforementioned experimental result ${ }^{7}$, suggesting that pores not only are unnecessary, but may in some cases be in contradiction with the experiment. Note also that in close proximity to the membrane phosphorous atoms water may bepresent at a density inferior to $1 \mathrm{~kg} / \mathrm{m}^{3}$, likely enough to donate a proton back to the peptide when it reaches the opposite leaflet, reconstituting its initial $\mathrm{Q}=+8 \mathrm{e}$ state in water solution. Fig. 9(center) plots the configuration corresponding to the spontaneous location that $\mathrm{R} 8$ reaches after $\sim 1 \mu \mathrm{s}$.
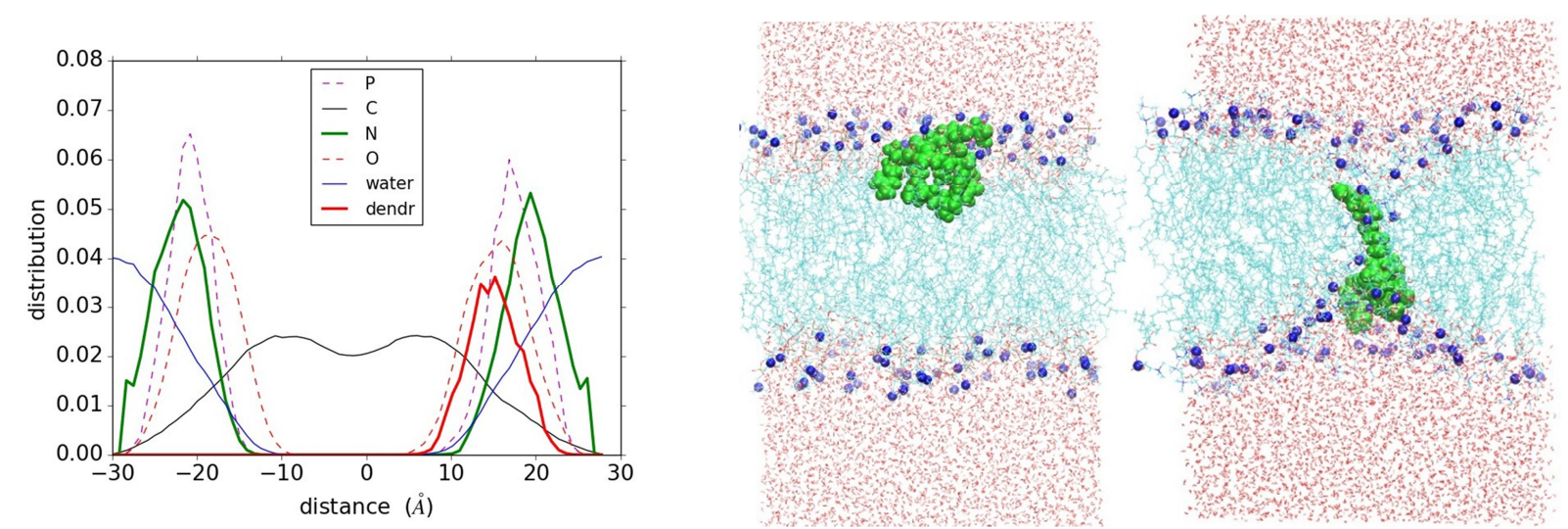

Fig. 9 (Left) Density profile of membrane, water, and the R8 dendrimer averaged over the simulation period of Fig.5(left); (Middle) MD snapshot at $1 \mu \mathrm{s}$ from the simulation in Fig.5(left). In blue the nitrogen atoms of the DOPC membrane. (Right) MD snapshot of a membrane bridging structure (discussed further below). 
Within this framework, we analyze now the second experiment related to the difficulty for hydrophilic drugs to partition inside membranes ${ }^{78}$, and to the necessity of chemical enhancers ${ }^{53}$. We examine whether our model can provide insight into apparent CPP behaviour as transdermal permeation enhancers, helping small drugs penetrating the skin barrier and reach the target site ${ }^{79}$. Without peptides the drug diffusion is driven by the gradient between the higher drug concentration and the target. This is a slow process and skin penetration enhancement techniques focus on increasing the drug flux via the transdermal route ${ }^{53,79}$.

The experiment examined enhancement of 5-FU permeation by three different types of arginine terminated cationic peptide dendrimers ${ }^{53}$, with charges $4^{+}, 8^{+}$, and $16^{+}$, named R4, R8, and R16. It employed human skin excised epidermis mounted on a support, so as to separate the donor and the receptor compartments ${ }^{53}$, with the donor chamber containing a saturated 5-FU drug solution with varying concentration of peptides. Of the three dendrimers tested, $\mathrm{R} 8$ bearing $8^{+}$showed the highest $5 F U$ flux, or cumulative amount of drug permeated, and the greatest amount of 5FU retained in human $\operatorname{skin}^{53}$. Without the presence of chemical penetration enhancers, it was shown that topical applications of $5 \mathrm{FU}$ show poor permeability ${ }^{80}$. Outstanding questions from the findings of the study include why the dendrimers enhance permeability; and why R8 is better than R4 and R16? Nonselective pores, when large enough, predict an equally likely passage of R4, R8, and R16, and when not large enough should favour R4 over R8, and R8 over R16. The experimental outcome shows that the highest flux is attained with R8, and, interestingly, even slightly better with octaarginine, which possesses an identical charge and very similar size, albeit with a linear architecture. Hence, the experiment cannot be explained with one of the pore models. As outlined in the introduction, we will argue here, via MD results, that one consequence of our proposed deprotonation scheme leads to a natural explanation of the 5-FU transdermal delivery experiment, whereas it is not clear that current poremodels are compatible with the experimental trend ${ }^{53}$.

Implementing a scheme for incremental movement of the centre of mass, coupled with equilibration after each movement, we dragged the peptide R8 from water to the centre of the membrane. When one of its two ends reaches the opposite leaflet, a bridge formation is favoured, i.e., the peptide connects the two leaflets. Fig. 9(right) shows the structure of the membrane after a peptide has been dragged inside the membrane. Note how this induced translocation or "dragging" procedure alters significantly the planarity of the membrane. This may be an unavoidable artifact of the MD procedure used to create the starting bridge structure prior to the unconstrained simulations. We tried to minimize this error, picking up one single atom located at the edge of one 
of its principal axis, to reduce the surface area of contact with the membrane during the movement into the core, and dragging slowly. This is the reason why we computed the PMF starting from the position when the peptide is already inside the membrane, that is, the position as depicted in Fig. 9(center), which is more realistic for the present case than the standard procedure since the peptide reaches that location spontaneously. Alternatively, the leaflet distortion observed in Fig. 9(right) may in fact be a consequence of the R8 peptide being slightly too short to create a bridge without significant membrane surface perturbation. Since the experiments ${ }^{53}$ utilized only R4, R8 and R16 peptides, we have not yet further explored this possibility.

After being in contact both leaflets, R8 is then left unconstrained in the simulations and we note that the peptide retains its perpendicular bridging conformation during the whole free energy calculation with no other constraining force applied to keep the peptide in place. This bridge stability can be ascribed to the electrostatic interactions between the side chain protonated guanidinium groups of the $\mathrm{Arg}$ with the $\mathrm{PO}_{4}{ }^{-}$groups of the phospholipid ${ }^{81,82}$, which form strong hydrogen bonds. In our MD simulations, seven 5-FU molecules are present in the water solution. Once the peptide is brought into its bridge configuration and thereafter left unconstrained, we noted that on both sides of the membrane 5-FU molecules enter the membrane, diffusing close to the peptide, sensing the locally hydrophilic environment there. We simulated octaarginine and R8 bridges with 5-FU in solution and - remarkably for the finite simulation times achievable - we were able to observe one case where a single 5-FU entered the membrane on one side, migrated stochastically along the peptide bridge and emerged to the opposite side after $800 \mathrm{~ns}$. This constitutes a 5-FU non-disruptive translocation event which did not require the presence of a pore. In fact, it is the first such instance of peptide-mediated drug translocation without endocytosis or pore formation that has been captured by atomistic MD simulations. In Fig. 10 we plot four snapshots taken across $700 \mathrm{~ns}$, from when the 5-FU molecule start interacting with the peptide, to the instant when 5-FU abandons the membrane from the other side, performing a stochastic crossmembrane migration during the journey. 


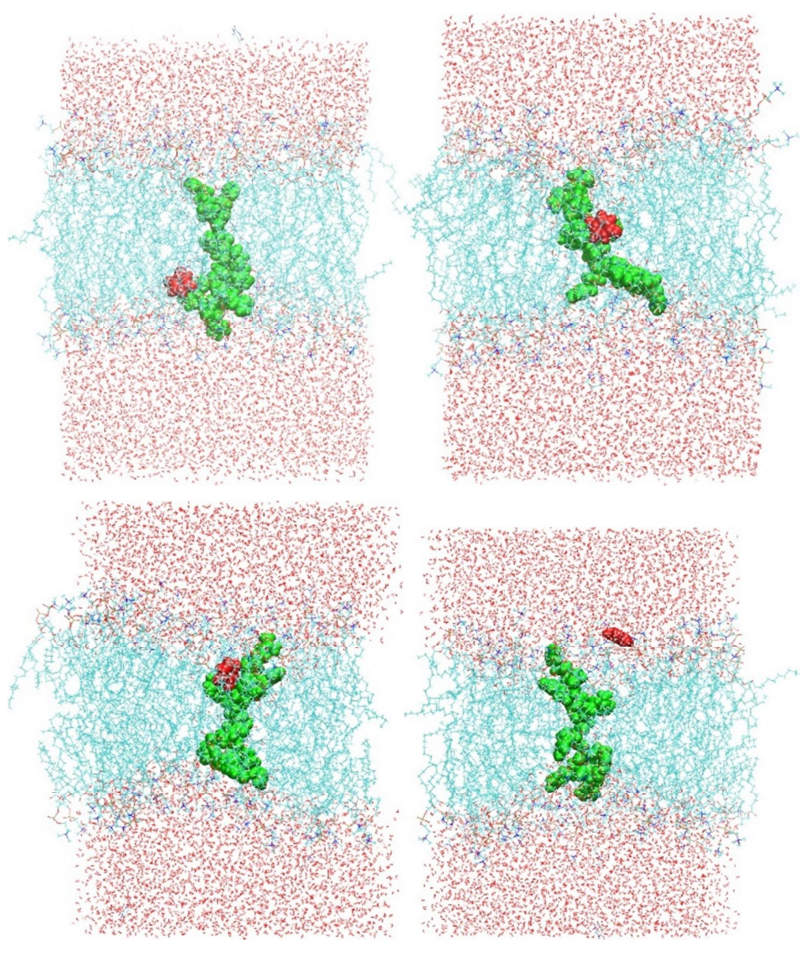

Fig. 10 5-Fluorouracil (red) crossing the membrane, as reported in four selected snapshots, from the beginning, top left, to the last frame when 5-FU completely crosses the membrane, bottom right. The peptide is green, the DOPC membrane in cyan, with water surrounding the membrane.

We have also simulated 5-FU in the presence of bridged peptides with a few different trial protonation states, for example with a peptide deprotonated only in the membrane core but not at the surfaces. 5-FU enters and rolls along the peptide easily. While no complete translocation events were observed in these cases, we observed several 5-FU penetrations up to the center of the membrane. We conclude that while the peptide protonation state is important (i) in facilitating membrane penetration and thereby potentially bridge formation; and (ii) once formed, contributing to stability of the bridge through electrostatic stabilization in the leaflet regions as noted above (effectively "anchoring" both ends of the bridge); the passage of 5-FU is facile regardless of peptide protonation state. To exemplify more quantitatively how easy it is for 5-FU to penetrate the membrane in presence of the peptide, we computed the PMF of 5-FU translocation across the membrane, with the reaction coordinate set as for the peptide, and with identical setup. Fig. 11 plots the result, comparing the PMF of 5-FU translocation without peptide (black profile) and with peptide (magenta). The reaction coordinate ( $\mathrm{d}$ in the picture) 


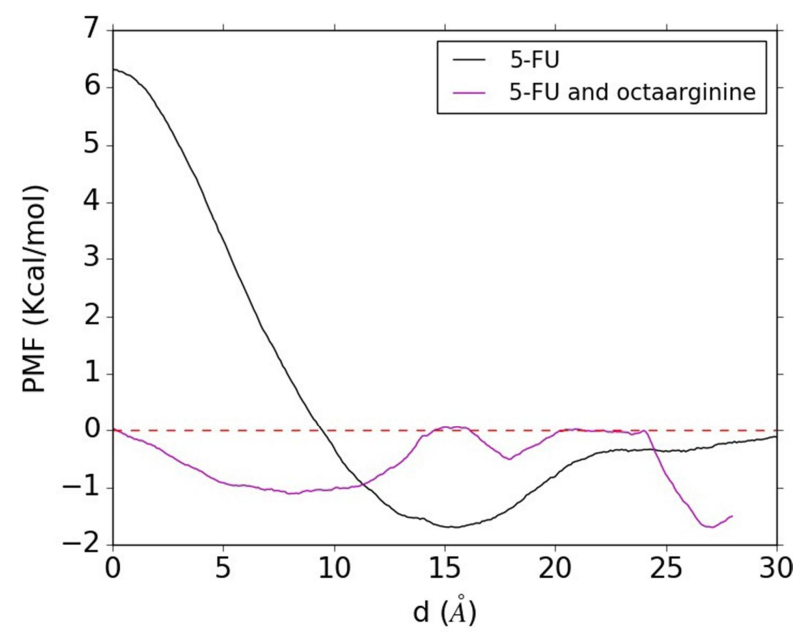

Fig. 11 (Black) PMF for 5-FU translocation across the membrane bilayer. The reaction coordinate is traced along the perpendicular axis of the bilayer. The distance $\mathrm{d}=0$ on the $\mathrm{x}$-axis corresponds to the center of the membrane. (Magenta) The PMF for 5-FU translocation across the membrane in the presence of an unrestrained bridging peptide. The 5-FU is initially positioned in the appropriate window, close to the peptide at around $0.8 \mathrm{~nm}$.

corresponds to the distance between the 5FU center of mass and the center of the membrane, projected along the $\mathrm{z}$-axis. The distance $\mathrm{d}=0$ corresponds to the membrane center. The PMF is computed with 15 windows of $2 \AA$, neglecting the first 800 samples and averaging around $1-1.5$ $\times 10^{6}$ samples per bin. We calculated the PMF of charged octaarginine here rather than R8 because we observed a translocation event with octaarginine only. For translocation of the 5-FU alone, the PMF maximum is calculated to be around $7 \mathrm{kcal} / \mathrm{mol}$. This is similar to a free energy computation of 5-FU across a DMPC bilayer, which was $\sim 10 \mathrm{kcal} / \mathrm{mol}^{83}$. In the presence of the bridging peptide, however, the PMF is zero or negative, suggesting that 5-FU traverses the membrane without facing any resistance. The probability to observe a translocation event in molecular dynamics simulations is prohibitively low, due to the limited spatial and time scales currently explorable with $\mathrm{MD}, \mu$ s and tens of nanometers, necessary to overcome the high energetic barrier posed by the membrane hydrophobic core to hydrophilic molecules. The fact that we captured such an event (Fig. 10) is clearly directly related to the almost complete removal of the activation barrier for translocation by the presence of the peptide bridge. This result is important in that it provides a simple explanation about how CPPs can enhance the permeation of 5-FU or indeed other small drug molecules through membranes. Our proposal also provides a simple explanation of the experimental observation that R8 provides a better enhancement of 5-FU permeability compared to R4 and R16 ${ }^{53}$. Quite simply, the R8 length matches the membrane thickness and is better 
optimized for bridge formation. R4 is too small to achieve a bridge; while R16 would involve more membrane distortion due to its size and so would be less likely to achieve a functional bridge structure.

\section{Discussion}

In this work we have proposed a model for non-invasive, non-endocytotic membrane translocation by cell penetrating peptides based on the potential ability of CPPs, which are positively charged in an aqueous environment, to shed excess protons via proton chain transfer as they experience a rapid decrease in water concentration and concomitant increase in hydrophobicity of the environment. This mechanism allows the peptides to penetrate the hydrophobic interior of the membrane with substantially lower free energy barriers. Our proposal does not reject the possibility that charged peptide residues might be stabilized within the membrane ${ }^{76}$, potentially via defect structures encompassing water molecules or membrane distortions. It is, however, appealing for its simplicity. While such membrane translocation by a peptide remains a rare event that we have not observed spontaneously on our $\mu \mathrm{s}$ MD timescale, the calculated PMF profiles clearly imply its feasibility. The calculated density profiles based on our simulations are in accord with recent neutron lamellar diffraction investigations of the interaction between a neutral DOPC membrane and TAT peptides ${ }^{7}$, which are inconsistent with pore formation mechanisms. We note that the chameleonic shedding of protons does offer up the question of whether such a local increase in hydrated proton density in the membrane leaflet region has implications for stability of the membrane structure. This question, however, will require substantial additional exploration as a part of further work.

Our MD simulations also reveal the potential for stable bridging structures in which the peptide spans the membrane. This stability is observed for both charged and non-charged bridging peptide structures. Such structures are seen to facilitate small drug molecule transport across the membrane, mediated by interactions between the drug and the bridging peptide. This offers the only current rationalization of recent experimental results revealing size-dependence in the enhancement afforded by short peptides for transdermal delivery of the anticancer drug $5 \mathrm{FU}^{53}$. We have observed an explicit trans-membrane delivery event - mediated by a bridging octaargenine peptide - of a 5FU anticancer drug during a single MD simulation, the first such demonstration via MD of noninvasive, non-endocytotic delivery of a small drug molecule. 
The proposed chameleon model for membrane translocation by small peptides - incorporating reversible proton shedding mediated through proton chain transfer - offers a new area of investigation of non-invasive, non-endocytotic cell penetration pathways.

\section{Acknowledgement}

Computational resources were provided by the National Computational Infrastructure (NCI Australia) facility at the Australian National University through the National Computational Merit Allocation Scheme; as well as CSC IT Centre for Science in Finland. We are grateful to Associate Professor Haibo Yu, University of Wollongong, for helpful discussions.

\section{Author contributions}

S.D.L. and S.C.S. designed the research, S.D.L. and P.S. performed the calculations, S.D.L., P.S., and S.C.S. analyzed the data and wrote the paper. All authors discussed the results and provided input for the manuscript. 


\section{References}

1. Jefferies, D., Hsu, P.-C. \& Khalid, S. Through the lipopolysaccharide glass: a potent antimicrobial peptide induces phase changes in membranes. Biochemistry 56, 1672-1679 (2017)

2. Lam, S. J. et al. Combating multidrug-resistant gram-negative bacteria with structurally nanoengineered antimicrobial peptide polymers. Nature Microbiology 1, 16162 (2016).

3. Hancock, R. E. Peptide antibiotics. The Lancet 349, 418-422 (1997).

4. Zhu, W. L. \& Shin, S. Y. Antimicrobial and cytolytic activities and plausible mode of bactericidal action of the cell penetrating peptide Penetratin and its Lys-linked twostranded peptide. Chemical biology \& drug design 73, 209-215 (2009).

5. Endoh, T. \& Ohtsuki, T. Cellular siRNA delivery using cell-penetrating peptides modified for endosomal escape. Advanced drug delivery reviews 61, 704-709 (2009).

6. Tünnemann, G. et al. Live-cell analysis of cell penetration ability and toxicity of oligoarginines. Journal of Peptide Science 14, 469-476 (2008).

7. Chen, X. et al. Efficient internalization of TAT peptide in zwitterionic DOPC phospholipid membrane revealed by neutron diffraction. BBA - Biomembranes 1859, 910-916 (2017).

8. Brock, R. The uptake of arginine-rich cell-penetrating peptides: putting the puzzle together. Bioconjugate Chemistry 25, 863-868 (2014).

9. Schow, E. V. et al. Arginine in membranes: the connection between molecular dynamics simulations and translocon-mediated insertion experiments. J. Memb. Biol. 239, 35-48 (2011).

10. Duchardt, F., Fotin-Mleczek, M., Schwarz, H., Fischer, R. \& Brock, R. A comprehensive model for the cellular uptake of cationic cell-penetrating peptides. Traffic 8, 848-866 (2007).

11. Fennouri, A., Mayer, S. F., Schroeder, T. B. \& Mayer, M. Single channel planar lipid bilayer

recordings of the melittin variant MelP5. BBA-Biomembranes 1859, 2051-2057 (2017).

12. Li, Z.-1., Ding, H.-m. \& Ma, Y.-q. Interaction of peptides with cell membranes: insights from molecular modeling. J. Phys: Cond. Mat. 28, 083001 (2016).

13. Leontiadou, H., Mark, A. E. \& Marrink, S. J. Antimicrobial peptides in action. J. Am. Chem. Soc. 128, 12156-12161 (2006).

14. Yesylevskyy, S., Marrink, S.-J. \& Mark, $\mathrm{A}_{23}$ E. Alternative mechanisms for the interaction of the cell-penetrating peptides penetratin and the TAT peptide with lipid bilayers. 
Biophys. J. 97, 40-49 (2009).

15. Woo, S. Y. \& Lee, H. Aggregation and insertion of melittin and its analogue MelP5 into lipid bilayers at different concentrations: effects on pore size, bilayer thickness and dynamics. Phys. Chem. Chem. Phys. 19, 7195-7203 (2017).

16. Li, Z.-1., Ding, H.-m. \& Ma, Y.-q. Translocation of polyarginines and conjugated nanoparticles across asymmetric membranes. Soft Matter 9, 1281-1286 (2012).

17. Marrink, S. J., Lindahl, E., Edholm, O. \& Mark, A. E. Simulation of the spontaneous aggregation of phospholipids into bilayers. J. Am. Chem. Soc. 123, 8638-8639 (2001).

18. Sun, D., Forsman, J., Lund, M. \& Woodward, C. E. Effect of arginine-rich cell penetrating peptides on membrane pore formation and life-times: a molecular simulation study. Phys. Chem. Chem. Phys. 16, 20785-20795 (2014).

19. Herce, H. D. \& Garcia, A. E. Molecular dynamics simulations suggest a mechanism for translocation of the HIV-1 TAT peptide across lipid membranes. Proc. Nat. Acad. Sci. 104, 20805-20810 (2007).

20. Ambroggio, E. E., Separovic, F., Bowie, J. H., Fidelio, G. D. \& Bagatolli, L. A. Direct visualization of membrane leakage induced by the antibiotic peptides: maculatin, citropin, and aurein. Biophys. J. 89, 1874-1881 (2005).

21. He, K., Ludtke, S. J., Huang, H. W. \& Worcester, D. L. Antimicrobial peptide pores in membranes detected by neutron in-plane scattering. Biochemistry 34, 15614-15618 (1995).

22. Allende, D., Simon, S. \& McIntosh, T. J. Melittin-induced bilayer leakage depends on lipid material properties: evidence for toroidal pores. Biophys. J. 88, 1828-1837 (2005).

23. Mihajlovic, M. \& Lazaridis, T. Antimicrobial peptides in toroidal and cylindrical pores. BBA-Biomembranes 1798, 1485-1493 (2010).

24. Gurnev, P. A., Yang, S.-T., Melikov, K. C., Chernomordik, L. V. \& Bezrukov, S. M. Cationic cell-penetrating peptide binds to planar lipid bilayers containing negatively charged lipids but does not induce conductive pores. Biophys. J. 104, 1933-1939 (2013).

25. Ter-Avetisyan, G. et al. Cell entry of arginine-rich peptides is independent of endocytosis. J. Biol. Chem. 284, 3370-3378 (2009).

26. Henriques, S. T., Melo, M. N. \& Castanho, M. A. Cell-penetrating peptides and antimicrobial peptides: how different are they? Biochem. J. 399, 1-7 (2006).

27. Akabori, K. et al. HIV-1 Tat membrane interactions probed using X-ray and neutron scattering, CD spectroscopy and MD simulations. BBA-Biomembranes 1838, 3078-3087 (2014).

28. Bennett, W. D., Hong, C. K., Wang, Y. \& Tieleman, D. P. Antimicrobial peptide 
simulations and the influence of force field on the free energy for pore formation in lipid bilayers. J. Chem. Theor. Comput. 12, 4524-4533 (2016).

29. Sengupta, D., Leontiadou, H., Mark, A. E. \& Marrink, S.-J. Toroidal pores formed by antimicrobial peptides show significant disorder. BBA-Biomembranes 1778, 2308-2317 (2008).

30. Chen, R. \& Mark, A. E. The effect of membrane curvature on the conformation of antimicrobial peptides: implications for binding and the mechanism of action. Euro Biophys. J. 40, 545-553 (2011).

31. Fretz, M. M. et al. Temperature-, concentration-and cholesterol-dependent translocation of L-and D-octa-arginine across the plasma and nuclear membrane of CD34+ leukaemia cells. Biochem. J. 403, 335-342 (2007).

32. Strandberg, E. \& Ulrich, A. S. NMR methods for studying membrane-active antimicrobial peptides. Concepts in Magnetic Resonance Part A 23, 89-120 (2004).

33. Phillips, J. C. et al. Scalable molecular dynamics with NAMD. J. Comput Chem. 26, 17811802 (2005).

34. Best, R. B. et al. Optimization of the additive CHARMM all-atom protein force field targeting improved sampling of the backbone $\varphi, \psi$ and side-chain $\chi_{1}$ and $\chi_{2}$ dihedral angles. J. Chem. Theor. Comput. 8, 3257-3273 (2012).

35. Wu, E. L. et al. CHARMM-GUI membrane builder toward realistic biological membrane simulations. J. Comput. Chem. 35, 1997-2004 (2014).

36. MacKerell Jr, A. D. et al. All-atom empirical potential for molecular modeling and dynamics studies of proteins. J. Phys. Chem. B 102, 3586-3616 (1998).

37. MacKerell, A. D. Jr, Feig, M., Brooks, C. L. III. Extending the treatment of backbone energetics in protein force fields: limitations of gas-phase quantum mechanics in reproducing protein conformational distributions in molecular dynamics simulations. $J$. Comput. Chem. 25, 1400-1415 (2004).

38. Kresten, L.-L., Nikola, T., Paul, M., Stefano, P. \& David, E. S. Structure and dynamics of an unfolded protein examined by molecular dynamics simulation. J. Am. Chem. Soc. 134, 3787-3791 (2012).

39. Jeffery, B. K. et al. Update of the CHARMM all-atom additive force field for lipids: validation on six lipid types. J. Phys. Chem. B 114, 7830-7843 (2010).

40. Jo, S., Kim, T., Iyer, V. G. \& Im, W. CHARMM-GUI: a web-based graphical user interface for CHARMM. J. Comput. Chem.229, 1859-1865 (2008). 
41. Venable, R. M. et al. CHARMM all-atom additive force field for sphingomyelin: elucidation of hydrogen bonding and of positive curvature. Biophys. J. 107, 134-145 (2014).

42. Jumin, L. et al. CHARMM-GUI input generator for NAMD, GROMACS, AMBER, OpenMM, and CHARMM/OpenMM simulations using the CHARMM36 additive force field. J. Chem. Theory Comput. 12, 405-413 (2016).

43. Jo, S., Lim, J. B., Klauda, J. B. \& Im, W. CHARMM-GUI membrane builder for mixed bilayers and its application to yeast membranes. Biophys. J. 97, 50-58 (2009).

44. Jo, S., Kim, T. \& Im, W. Automated builder and database of protein/membrane complexes for molecular dynamics simulations. PLoS ONE 2, e880 (2007).

45. Vanommeslaeghe, $\mathrm{K}$. et al. CHARMM general force field (CGenFF): a force field for drug-like molecules compatible with the CHARMM all-atom additive biological force fields. J. Comput. Chem. 31, 671-690 (2010).

46. Vanommeslaeghe, K. \& MacKerell, A. D. Jr. Automation of the CHARMM general force field (CGenFF) I: bond perception and atom typing. J. Chem. Info. Model. 52, 3144-3154 (2012).

47. Vanommeslaeghe, K., Raman, E. P. \& MacKerell, A. D. Jr. Automation of the CHARMM general force field (CGenFF) II: assignment of bonded parameters and partial atomic charges. J. Chem. Info. Model. 52, 3155-3168 (2012).

48. www.ks.uiuc.edu/Training/Tutorials/namd-index.html.

49. De Luca, S. et al. Dynamical interactions of 5-fluorouracil drug with dendritic peptide vectors: the impact of dendrimer generation, charge, counterions, and structured water. $J$. Phys. Chem. B 120, 5732-5743 (2016).

50. De Luca, S., Chen, F., Seal, P., Stenzel, M. H. \& Smith, S. C. Binding and release between polymeric carrier and protein drug: pH-mediated interplay of coulomb forces, hydrogen bonding, van der Waals interactions, and entropy. Biomacromolecules 18, 3665-3677 (2017).

51. Maiti, P. K., Çă̌in, T.; Wang, G. \& Goddard III, W. A. Structure of PAMAM dendrimers: generations 1 through 11. Macromolecules 37, 6236-6254 (2004).

52. Mansfield, M. L. \& Jeong, M. Simulation of lattice dendrimers by a Monte Carlo technique with detailed balance. Macromolecules 35, 9794-9798 (2002).

53. Mutalik, S., Shetty, P. K., Kumar, A., Kalra, R. \& Parekh, H. S. Enhancement in deposition and permeation of 5-fluorouracil through hyman epidermis assisted by peptide dendrimers. Drug Delivery 21, 44-54 (2014). 
54. Martínez, L., Andrade, R., Birgin, E. G., Martínez, J. M. Packmol: a package for building initial configurations for molecular dynamics simulations. J. Comput Chem. 30, 2157-2164 (2009).

55. Humphrey, W., Dalke, A., Schulten, K. VMD: Visual Molecular Dynamics. J. Mol. Graph. 14, 33-38 (1996).

56. Jorgensen, W. L., Chandrasekhar, J., Madura, J. D., Impey, R. W. \& Klein, M. L. Comparison of simple potential functions for simulating liquid water. J. Chem. Phys. 79, 926-935 (1983).

57. Darden, T., York, D. \& Pedersen, L. Particle Mesh Ewald: an N $\log (\mathrm{N})$ method for Ewald sums in large systems. J. Chem. Phys. 98, 10089-10092 (1993).

58. Allen, M. P. \& Tildesley, D. J. Computer Simulation of Liquids.; Oxford University Press: New York, 1987.

59. Ulrich, A. S., Sami, M. \& Watts, A. Hydration of DOPC bilayers by differential scanning calorimetry. BBA-Biomembranes 1191, 225-230 (1994).

60. Helmut, H., Michael, S. \& Klaus, S. Molecular dynamics simulation of a bilayer of 200 lipids in the gel and in the liquid crystal phase. J. Phys. Chem. 97, 8343-8360 (1993).

61. Matsuzaki, K. Why and how are peptidelipid interactions utilized for self-defense? Magainins and tachyplesins as archetypes. BBA - Biomembranes 1462, 1-10 (1999).

62. Sharon, M. L. Molecular simulation of the transport of drugs across model membranes. $J$. Phys. Chem. Lett. 5, 1659-1665 (2014).

63. Darve, E., Rodrguez-Gmez, D. \& Pohorille, A. Adaptive biasing force method for scalar and vector free energy calculations. J. Chem. Phys. 128, 144120 (2008).

64. Hnin, J., Fiorin, G., Chipot, C. \& Klein, M. L. Exploring multidimensional free energy landscapes using time-dependent biases on collective variables. J. Chem. Theory Comput. 6, 35-47 (2010).

65. J. Tomasi, B. Mennucci, R. Cammi, Chem. Rev. 105, 2999 (2005).

66. Gaussian 16, Revision A.03, M. J. Frisch, G. W. Trucks, H. B. Schlegel, G. E. Scuseria, M. A. Robb, J. R. Cheeseman, G. Scalmani, V. Barone, G. A. Petersson, H. Nakatsuji, X. Li, M. Caricato, A. V. Marenich, J. Bloino, B. G. Janesko, R. Gomperts, B. Mennucci, H. P. Hratchian, J. V. Ortiz, A. F. Izmaylov, J. L. Sonnenberg, D. Williams-Young, F. Ding, F. Lipparini, F. Egidi, J. Goings, B. Peng, A. Petrone, T. Henderson, D. Ranasinghe, V. G. 27

Zakrzewski, J. Gao, N. Rega, G. Zheng, W. Liang, M. Hada, M. Ehara, K. Toyota, R. 
Fukuda, J. Hasegawa, M. Ishida, T. Nakajima, Y. Honda, O. Kitao, H. Nakai, T. Vreven, K. Throssell, J. A. Montgomery, Jr., J. E. Peralta, F. Ogliaro, M. J. Bearpark, J. J. Heyd, E. N. Brothers, K. N. Kudin, V. N. Staroverov, T. A. Keith, R. Kobayashi, J. Normand, K. Raghavachari, A. P. Rendell, J. C. Burant, S. S. Iyengar, J. Tomasi, M. Cossi, J. M. Millam, M. Klene, C. Adamo, R. Cammi, J. W. Ochterski, R. L. Martin, K. Morokuma, O. Farkas, J. B. Foresman, D. J. Fox, Gaussian, Inc., Wallingford CT, 2016.

67. Sun, D., Forsman, J. \& Woodward, C. E. Evaluating force fields for the computational prediction of ionized arginine and lysine side-chains partitioning into lipid bilayers and octanol. J. Chem. Theory Comput. 11, 1775-1791 (2015).

68. Takechi-Haraya, Y. et al. Glycosaminoglycan binding and non-endocytic membrane translocation of cell-permeable octaarginine monitored by real-time in-cell NMR spectroscopy. Pharmaceuticals 10, 42 (2017).

69. Li, L., Vorobyov, I., MacKerell, A. D. \& Allen, T. W. Is arginine charged in a membrane? Biophys. J. 94, L11-L13 (2008).

70. Harms, M. J., Schlessman, J. L., Sue, G. R. \& Bertrand García-Moreno, E. Arginine residues at internal positions in a protein are always charged. Proc. Natl. Acad. Sci. USA 108, 18954 18959 (2011).

71. Hristova, K. \& Wimley, W. C. A look at arginine in membranes. J. Memb. Biol. 239, 49-56 (2011).

72. Li, L., Vorobyov, I. \& Allen, T. W. The different interactions of lysine and arginine side chains with lipid membranes. J. Phys. Chem. B 117, 11906-11920 (2013).

73. Bouchet, A., Lairion, F. \& Disalvo, E. A. Role of guanidinium group in the insertion of 1arginine in $\{$ DMPE $\}$ and $\{$ DMPC $\}$ lipid interphases. BBA - Biomembranes 1798, 616-623 (2010).

74. MacCallum, J. L., Bennett, W. D. \& Tieleman, D. P. Distribution of amino acids in a lipid bilayer from computer simulations. Biophys. J. 94, 3393-3404 (2008).

75. Yoo, J. \& Cui, Q. Does arginine remain protonated in the lipid membrane? Insights from microscopic pKa calculations. Biophys. J. 94, L61-L63 (2008).

76. S. Wang, S. C. Smith, Phys. Chem. Chem. Phys. 9, 452 (2007).

77. Chen, X. et al. Efficient internalization of TAT peptide in zwitterionic DOPC phospholipid membrane revealed by neutron diffraction. ${ }_{2} B A$-Biomembranes 1859, 910-916 (2017).

78. Kope, W., Telenius, J. \& Khandelia, H. Molecular dynamics simulations of the interactions 
of medicinal plant extracts and drugs with lipid bilayer membranes. FEBS Journal 280, 2785-2805 (2013).

79. Stanley, S. Transdermal drug delivery: past, present, future. Molecular Interventions 4, 308$312(2005)$.

80. Singh, B. \& Jayaswal, S. Iontophoretic delivery of 5-fluorouracil through excised human stratum corneum. Drug discoveries \& therapeutics 2, 128-135 (2008).

81. Li, J. et al. Molecular simulations suggest how a branched antimicrobial peptide perturbs a bacterial membrane and enhances permeability. BBA-Biomembranes 1828, 1112-1121 (2013).

82. Costa, P. J., Marques, I. \& Flix, V. Interaction of a calix[4]arene derivative with a \{DOPC\} bilayer: biomolecular simulations towards chloride transport. BBA - Biomembranes $\mathbf{1 8 3 8}$, 890-901 (2014).

83. Khajeh, A. \& Modarress, H. Effect of cholesterol on behavior of 5-fluorouracil (5-FU) in a DMPC lipid bilayer, a molecular dynamics study. Biophys. Chem. 187, 43-50 (2014). 


\section{$\underline{\text { Supporting Information }}$}

Cell membrane penetration without pore formation: chameleonic properties of dendrimers in response to hydrophobic and hydrophilic environments

Sergio de Luca ${ }^{1}$, Prasenjit Seal ${ }^{2}$, Harendra S. Parekh ${ }^{3}$, Karnaker R Tupally ${ }^{3}$ \& Sean C. Smith ${ }^{1, *}$

${ }^{1}$ Integrated Materials Design Laboratory, Department of Applied Mathematics, Research School of Physics, Australian National University, Acton, ACT 2601, Australia.

${ }^{2}$ Department of Chemistry, University of Helsinki, P.O. Box 55 (A.I. Virtasen aukio 1), Helsinki 00014, Finland.

${ }^{3}$ School of Pharmacy, The University of Queensland, Brisbane, QLD 4072, Australia.

E-mail: sean.smith@anu.edu.au 

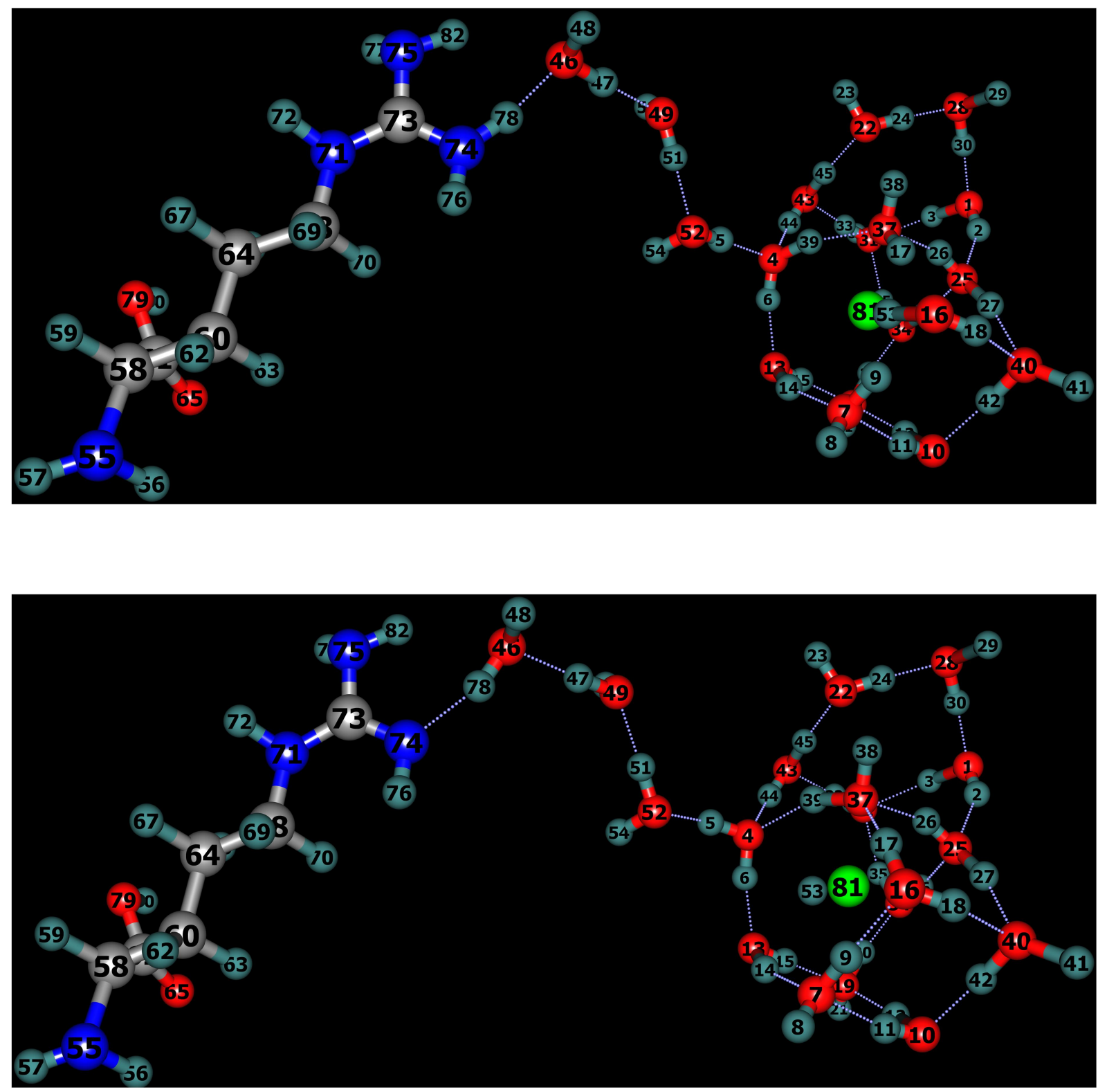

Fig. S1: The complete figures of the proton transfer pathway. Top: The initial structure, scan point 1, Bottom: the final structure, scan point 14. 
Table S1: Bond distances of each scanning points in the PTP curve along with their electronic energies at B3LYP/6-31G(d) level of theory. The yellow highlighted portion are the increments done for each bonds in our adopted strategy. The mechanism is PTP-PUSH.

\begin{tabular}{ccccccccc}
\hline \multirow{2}{*}{$\begin{array}{c}\text { Scan } \\
\text { points }\end{array}$} & \multicolumn{9}{c}{ Bond lengths $(\AA)$} & \multirow{3}{*}{ Energies (a.u.) } \\
\cline { 2 - 7 } & $\mathrm{N}_{74}-\mathrm{H}_{78}$ & $\mathrm{O}_{46}-\mathrm{H}_{47}$ & $\mathrm{O}_{49}-\mathrm{H}_{51}$ & $\mathrm{O}_{52}-\mathrm{H}_{5}$ & $\mathrm{O}_{4}-\mathrm{H}_{39}$ & $\mathrm{O}_{37}-\mathrm{H}_{17}$ & $\mathrm{O}_{16}-\mathrm{H}_{53}$ & \\
\hline 1 & 1.03 & 0.99 & 0.98 & 0.98 & 0.98 & 0.98 & 0.99 & -2442.8437017 \\
2 & 1.18 & 0.99 & 0.98 & 0.98 & 0.98 & 0.98 & 0.99 & -2442.8386497 \\
3 & 1.33 & 1.14 & 0.98 & 0.98 & 0.98 & 0.98 & 0.99 & -2442.8252374 \\
4 & 1.48 & 1.29 & 1.13 & 0.98 & 0.98 & 0.98 & 0.99 & -2442.8131362 \\
5 & 1.63 & 1.44 & 1.28 & 1.13 & 0.98 & 0.98 & 0.99 & -2442.8137444 \\
6 & 1.73 & 1.59 & 1.43 & 1.28 & 1.13 & 0.98 & 0.99 & -2442.8144453 \\
7 & 1.73 & 1.69 & 1.58 & 1.43 & 1.28 & 1.13 & 0.99 & -2442.8060953 \\
8 & 1.73 & 1.69 & 1.68 & 1.58 & 1.43 & 1.28 & 1.19 & -2442.7970493 \\
9 & 1.73 & 1.69 & 1.68 & 1.73 & 1.58 & 1.43 & 1.39 & -2442.814036 \\
10 & 1.73 & 1.69 & 1.68 & 1.73 & 1.73 & 1.63 & 1.59 & -2442.8552341 \\
11 & 1.73 & 1.69 & 1.68 & 1.73 & 1.73 & 1.78 & 1.89 & -2442.8940443 \\
12 & 1.73 & 1.69 & 1.68 & 1.73 & 1.73 & 1.78 & 2.19 & -2442.9062212 \\
\hline
\end{tabular}

Table S2: Bond distances of each scanning points in the PTP curve along with their electronic energies at B3LYP/6-31G(d) level of theory. The yellow highlighted portion are the increments done for each bonds in our adopted strategy. The mechanism is PTP-PULL.

\begin{tabular}{ccccccccc}
\hline \multirow{2}{*}{$\begin{array}{c}\text { Scan } \\
\text { points }\end{array}$} & \multicolumn{9}{c}{ Bond lengths $(\AA)$} \\
\cline { 2 - 7 } & $\mathrm{N}_{74}-\mathrm{H}_{78}$ & $\mathrm{O}_{46}-\mathrm{H}_{47}$ & $\mathrm{O}_{49}-\mathrm{H}_{51}$ & $\mathrm{O}_{52}-\mathrm{H}_{5}$ & $\mathrm{O}_{4}-\mathrm{H}_{39}$ & $\mathrm{O}_{37}-\mathrm{H}_{17}$ & $\mathrm{O}_{16}-\mathrm{H}_{53}$ & \\
\hline 1 & 1.70 & 1.68 & 1.69 & 1.77 & 1.77 & 1.81 & 2.43 & -2442.9105898 \\
2 & 1.70 & 1.68 & 1.69 & 1.77 & 1.77 & 1.81 & 2.13 & -2442.9068129 \\
3 & 1.70 & 1.68 & 1.69 & 1.77 & 1.77 & 1.66 & 1.83 & -2442.8811249 \\
4 & 1.70 & 1.68 & 1.69 & 1.77 & 1.62 & 1.51 & 1.53 & -2442.8325058 \\
5 & 1.70 & 1.68 & 1.69 & 1.62 & 1.47 & 1.36 & 1.23 & -2442.7966 \\
6 & 1.70 & 1.68 & 1.54 & 1.47 & 1.32 & 1.16 & 0.99 & -2442.8000316 \\
7 & 1.70 & 1.53 & 1.39 & 1.32 & 1.17 & 0.98 & 0.99 & -2442.8051053 \\
8 & 1.55 & 1.38 & 1.24 & 1.17 & 0.98 & 0.98 & 0.99 & -2442.8081477 \\
9 & 1.40 & 1.23 & 1.09 & 0.98 & 0.98 & 0.98 & 0.99 & -2442.8171168 \\
10 & 1.25 & 1.08 & 0.98 & 0.98 & 0.98 & 0.98 & 0.99 & -2442.8326068 \\
11 & 1.15 & 0.99 & 0.98 & 0.98 & 0.98 & 0.98 & 0.99 & -2442.8405025 \\
12 & 1.03 & 0.99 & 0.98 & 0.98 & 0.98 & 0.98 & 0.99 & -2442.8438304 \\
\hline
\end{tabular}


Table S3: Bond distances of each scanning points in the PTP curve along with their electronic energies at B3LYP/6-31G(d) level of theory. The yellow highlighted portion are the increments done for each bonds in our adopted strategy. The mechanism is PTP-SYNC.

\begin{tabular}{ccccccccc}
\hline \multirow{2}{*}{$\begin{array}{c}\text { Scan } \\
\text { points }\end{array}$} & \multicolumn{7}{c}{ Bond lengths $(\AA)$} & \multirow{3}{*}{ Energies (a.u.) } \\
\cline { 2 - 7 } & $\mathrm{N}_{74}-\mathrm{H}_{78}$ & $\mathrm{O}_{46}-\mathrm{H}_{47}$ & $\mathrm{O}_{49}-\mathrm{H}_{51}$ & $\mathrm{O}_{52}-\mathrm{H}_{5}$ & $\mathrm{O}_{4}-\mathrm{H}_{39}$ & $\mathrm{O}_{37}-\mathrm{H}_{17}$ & $\mathrm{O}_{16}-\mathrm{H}_{53}$ & \\
\hline 1 & 1.03 & 0.99 & 0.98 & 0.98 & 0.98 & 0.98 & 0.99 & -2442.8437017 \\
2 & 1.18 & 1.14 & 1.13 & 1.13 & 1.13 & 1.13 & 1.19 & -2442.7924393 \\
3 & 1.33 & 1.29 & 1.28 & 1.28 & 1.28 & 1.28 & 1.39 & -2442.7314821 \\
4 & 1.48 & 1.44 & 1.43 & 1.43 & 1.43 & 1.43 & 1.59 & -2442.7513012 \\
5 & 1.63 & 1.59 & 1.58 & 1.58 & 1.58 & 1.63 & 1.89 & -2442.8483154 \\
6 & 1.73 & 1.69 & 1.68 & 1.73 & 1.73 & 1.78 & 2.19 & -2442.9062212 \\
\hline
\end{tabular}

